Preprint SB/F/05-333

\title{
The quantum algebra of superspace
}

\author{
N. Hatcher, A. Restuccia and J. Stephany \\ Universidad Simón Bolivar, Departamento de Física, Apartado Postal 89000, Caracas \\ 1080-A, Venezuela. \\ e-mail: nhatcher@fis.usb.ve, arestu@usb.ve,stephany@usb.ve
}

\begin{abstract}
We present the complete set of $N=1, D=4$ quantum algebras associated to massive superparticles. We obtain the explicit solution of these algebras realized in terms of unconstrained operators acting on the Hilbert space of superfields. These solutions are expressed using the chiral, anti-chiral and tensorial projectors which define the three irreducible representations of the supersymmetry on the superfields. In each case the space-time variables are non-commuting and their commutators are proportional to the internal angular momentum of the representation. The quantum algebra associated to the chiral or the anti-chiral projector is the one obtained by the quantization of the Casalbuoni-Brink-Schwarz (superspin 0 ) massive superparticle. We present a new superparticle action for the (superspin 1/2) case and show that their wave functions are the ones associated to the irreducible tensor multiplet.
\end{abstract}

\section{UNIVERSIDAD SIMON BOLIVAR}




\section{Introduction and summary}

The superparticles, the point-like objects which move in the Salam and Strathdee superspace 23] were first described by Casalbuoni 2] almost 30 years ago, and by Brink and Schwarz 3 soon afterwards. They have internal angular momentum and built in supersymmetry. As a simple model of a classical particle with internal angular momentum they bear some resemblance with the relativistic top studied by Hanson and Regge [4, but they also have novel interesting features of it own. The real interest in these models arose with the advent of the superstring action [5] and the understanding of the structure of the constraints that appear in the canonical analysis. Siegel [6] showed that the massless superparticle possess an extra local fermionic symmetry now called kappa symmetry which is also present in the massive case when central charges are included [7. This symmetry develop later in a guiding principle that was used to select valid Lagrangeans for supersymmetric systems. The superstring and the supermembrane actions were discovered imposing this symmetry. Also it was discovered that by imposing the kappa symmetry to the action of a supermembrane in a background field one obtains the whole set of eleven dimensional supergravity equations for the component fields.

But the virtues of supersymmetry and kappa symmetry are accompanied with problems. The canonical analysis of the massless superparticle shows that the set of first class constraints that generate kappa symmetry were tied to a set of second class constraints. With time it became evident that it was not possible to split this constraints in a covariant and irreducible way. Over the years many ways to deal with the problem of covariant quantization of the superparticle and superstring were proposed with different grades of success. Many of these methods needed to include a infinite tower of ghosts and the meaning of the resulting BRST operator became unclear to say the

least. Some other approaches were linked to the harmonic superspace, where the set of infinite fields are codified in an organized way [8]. Infinite fields appeared also in the formulations using twistor variables where as an additional complication several different versions of what a twistor should be have been proposed. Very interesting geometrical ideas related to twistor theory were inherent in the work of doubly supersymmetric particles and strings for which Siegel symmetry has been understood as a diffeomorphism of the superworld-line. Nevertheles, although, extensions for $\mathrm{D}=10$ using Lorentz harmonics [9], superembeddings techniques [10] and more recently pure spinors [1] have been proposed, the goal of constructing off-shell D=10 Super Yang Mills has not been achieved.

A related but quite different class of systems is that of massive superparticles. When central charges are absent they do not posses kappa symmetry and first and second class constraints are not mixed. The solution of the superparticle algebra of the observables of the theory has not been discussed in the literature. In this paper we solve this problem for $D=4$ and $N=1$ massive superparticles. We obtain the explicit solution of these algebras realized in terms of unconstrained operators acting on the Hilbert space of superfields. These solutions are expressed using the chiral, anti-chiral and tensorial projectors which define the three irreducible representations of the supersymmetry on the superfields.

This paper is organized as follows. In section 2 we discuss the classical dynamics 
of the massive superparticle which is subject to second class constraints. We present the algebra obtained using Dirac procedure. The resulting algebra of brackets [2] is not straightforward to implement quantum mechanically because the space time variables turns out to be non-commutative, $\left[x^{\mu}, x^{\nu}\right] \neq 0$. This problem may be circumvented by finding a reduced set of coordinates that satisfy canonical commutation relations which, not surprisingly, result to be the chiral coordinates as suggested by Casalbuoni [2]. In section 3 we construct the quantum algebra of the superparticle with operators acting on the Hilbert space of states corresponding to the irreducible representations of the supersymmetry. This is done with the aid of the projectors (chiral, anti-chiral and tensorial) to the three subspaces of the space of superfields which allow an irreducible representation of the supersymmetry algebra (associated respectively to the chiral, antichiral and irreducible tensor multiplets). We then obtain three well defined covariant operatorial solutions for the superparticle algebra, two with superspin 0 associated to the chiral or to the anti-chiral massive superparticle and one with super spin $1 / 2$ with should be associated to a different super particle. In each case we obtain the explicit operatorial expression for the internal angular momentum. The chiral and anti-chiral massive superparticles are described by the standard massive superparticle action, while the super spin 1/2 superparticle is described by a different action. In section 4 we present and analyze the action corresponding to the super spin $1 / 2$ superparticle. We show that the wave functions of this new superparticle are superfields projected by the tensorial projector which reduce them to the degrees of freedom of the irreducible tensor multiplet (of super spin 1/2). In section 5 we discuss the applicability of our results in a more general setup. Finally in section 6 we present our conclusion and outlook.

\section{Classical mechanics in the superspace}

Let us consider the standard massive superparticle in $D=4$. The metric signature is $\eta_{\mu \nu}=\operatorname{diag}\{-1,+1,+1,+1\}$ and the superspace coordinates are $\left(x^{\mu}, \theta^{a i}, \bar{\theta}^{\dot{a}}{ }_{i}\right)$, where $a=1,2$ is a spinor index and $i=1, \ldots, N$ is the number of supersymmetric charges. Naturally $\left(\theta^{a i}\right)^{*}=\bar{\theta}_{i}{ }_{i}$. We choose Dirac matrices to be off-diagonal and given by,

$$
\gamma^{\mu}=\left(\begin{array}{cc}
0 & \sigma^{\mu} \\
\bar{\sigma}^{\mu} & 0
\end{array}\right)
$$

with $\sigma_{a b}^{\mu}$ the Pauli matrices. The action principle for the superparticle is given by [2]

$$
S=\frac{1}{2} \int d \tau\left(e^{-1} \omega^{\mu} \omega^{\nu} \eta_{\mu \nu}-e m^{2}\right)
$$

where $\omega^{\mu}=\dot{x}^{\mu}-i \dot{\theta}^{a i} \sigma_{a \dot{b}}^{\mu} \bar{\theta}_{i}{ }_{i}+i \theta^{a i} \sigma_{a \dot{b}}^{\mu}{ }_{i}^{\dot{\theta^{b}}}{ }_{i}$ is defined for convenience. 
The generalized momenta are given by

$$
\begin{gathered}
\pi_{e}=0 \\
p_{\mu}=e^{-1} \omega_{\mu} \\
\pi_{a i}=-i p_{\mu} \sigma_{a \dot{b}}^{\mu} \bar{\theta}_{i}^{\dot{b}} \\
\bar{\pi}_{\dot{b}}^{i}=-i p_{\mu} \bar{\theta}^{a i} \sigma_{a \dot{b}}^{\mu} .
\end{gathered}
$$

and satisfy the canonical Poisson bracket relations [2]

$$
\begin{gathered}
\left\{x^{\mu}, p_{\nu}\right\}=\delta^{\mu}{ }_{\nu} \\
\left\{\theta^{a i}, \pi_{b j}\right\}=-\delta^{a}{ }_{b} \delta^{i}{ }_{j} .
\end{gathered}
$$

Along the conserved quantities related to Super Poincarè invariance the total angular momentum is given by

$$
\begin{gathered}
J_{\mu \nu}=L_{\mu \nu}+S_{\mu \nu} \\
L_{\mu \nu}=x_{\mu} p_{\nu}-x_{\mu} p_{\nu} \\
S_{\mu \nu}=-\frac{1}{4}\left(\theta^{a i} \sigma_{\mu \nu}{ }^{b} \pi_{b i}+\bar{\pi}_{\dot{a}}^{i} \bar{\sigma}_{\mu \nu} \dot{a}_{\dot{b}} \bar{\theta}^{b}{ }_{i}\right)
\end{gathered}
$$

For this system, there is only one first class constraint $\pi_{e}=0$ related to the reparametrization invariance of the action which implies the first class secondary constraint $p^{2}+m^{2}=0$. There appear also the constraints,

$$
\begin{gathered}
d_{a i} \equiv \pi_{a i}+i p_{\mu} \sigma_{a \dot{b}}^{\mu} \bar{\theta}_{i}^{\dot{b}}=0 \\
\bar{d}_{\dot{b}}^{i} \equiv \bar{\pi}_{\dot{b}}{ }^{i}+i p_{\mu} \theta^{a i} \sigma_{a b}^{\mu}=0 \\
\left\{d_{a i}, \bar{d}_{\dot{b}}^{j}\right\}=-i \delta_{i}^{j} p_{\mu} \sigma_{a \dot{b}}^{\mu} \equiv C_{a i b}{ }^{j} .
\end{gathered}
$$

which are second class since the matrix $C_{a i b}{ }^{j}$ is non singular if $m \neq 0$. The Dirac brackets are given by,

$$
\{F, G\}_{\mathrm{D}}=\{F, G\}-\left\{F, d_{a i}\right\} \hat{C}_{j}^{a i \dot{b}}\left\{\bar{d}_{\dot{b}}^{j}, G\right\}-\left\{F, \bar{d}_{\dot{b}}^{j}\right\} \hat{C}_{j}^{a i \dot{b}}\left\{d_{a i}, G\right\}
$$

Here $\hat{C}_{j}^{a i \dot{b}}$ is the matrix $\hat{C}_{j}^{a i \dot{b}}=\frac{1}{2 i p^{2}} p_{\mu} \bar{\sigma}^{\mu a \dot{b}} \delta_{j}^{i}$ and verifies

$$
C_{a i b}{ }^{j} \hat{C}_{j}^{b k \dot{b}}=\delta_{i}{ }^{i} \delta_{a}{ }^{b}
$$


Calculating the Dirac brackets for all coordinates and momenta the result is [2],

$$
\begin{gathered}
\left\{\theta^{a i}, \theta^{b j}\right\}_{\mathrm{D}}=\left\{\pi_{a i}, \pi_{b j}\right\}_{\mathrm{D}}=\left\{p_{\mu}, p_{\nu}\right\}_{\mathrm{D}}=0 \\
\left\{\theta^{a i}, \bar{\theta}^{\dot{a}}{ }_{j}\right\}_{\mathrm{D}}=\frac{-1}{2 i p^{2}} p_{\mu} \bar{\sigma}^{\mu \dot{a} a} \delta_{j}^{i} \\
\left\{x^{\mu}, \theta^{a i}\right\}_{\mathrm{D}}=\frac{1}{2 p^{2}} p_{\nu} \bar{\sigma}^{\nu \dot{a} a} \theta^{b i} \sigma_{b \dot{a}}^{\mu} \\
\left\{x^{\mu}, \bar{\theta}^{\dot{a}}{ }_{i}\right\}_{\mathrm{D}}=\frac{1}{2 p^{2}} p_{\nu} \bar{\sigma}^{\nu \dot{a} a} \bar{\theta}^{\dot{b}}{ }_{i} \sigma_{a \dot{b}}^{\mu} \\
\left\{x^{\mu}, x^{\nu}\right\}_{\mathrm{D}}=\frac{-S^{\mu \nu}}{p^{2}},
\end{gathered}
$$

Using (12), (13) and (A.4) the equation for the internal angular momentum $S^{\mu \nu}$ (11) may be written also in the form,

$$
S^{\mu \nu}=\varepsilon^{\mu \nu \rho \lambda} p_{\rho} \theta^{a i} \sigma_{\lambda a \dot{b}} \bar{\theta}_{i}^{\dot{b}}
$$

Before applying the quantization rules to the algebra above to construct the quantum theory of the superparticle one can first use a different approach which in particular identifies the Hilbert space associate to the action (2) . The second class constraints are separated in two subsets, the subset $d_{a}$ and its complex conjugate $\bar{d}_{\dot{a}}$. It has been proposed that in such a situation, if it is too complicated to impose all the constraints it may be sufficient to impose only one of the two sets because then the matrix elements are zero. That is in general, for constraints $\left(\phi_{\alpha}, \bar{\phi}_{\alpha}\right)$ imposing $\hat{\phi}_{\alpha}|V\rangle=0$ then $\langle V| \hat{\bar{\phi}}_{\alpha}=$ 0 . For the superparticle, this idea goes back to Casalbuoni 2 and has been applied extensively by Lusana 12, Frydryszak [13] and collaborators. Although the application of this approach in the cases considered by these authors provides the correct answer, the approach fails to work in general. When the method works correctly it can be understood as follows. For $\left\{\phi_{\alpha}, \bar{\phi}_{\beta}\right\}$ a set of second class constraints the measure in the corresponding functional integral is $[\operatorname{det}\{\phi, \bar{\phi}\}]^{1 / 2}$. In the particular case in which $\operatorname{det}\left\{\phi_{\alpha}, \phi_{\beta}\right\}=0$ then the above measure reduces to $\operatorname{det}\left\{\phi_{\alpha}, \bar{\phi}_{\beta}\right\}$ which is exactly the functional measure for a set of first class constraints $\phi_{\alpha}$ with a gauge fixing condition [14, 15]. In the case under study it is easily seen that $\bar{d}_{\dot{a}}$ alone are a set of first class constraints and thus the model is equivalent to a gauge system in which only this set of first class constraints exists. We are then free to choose a different gauge fixing condition. This method was applied in reference [16] to show that the wave functions of the $D=9$, $N=2$, massive superparticle with central charges expand a KKB ultrashort multiplet [17.

The canonical coordinates necessary to develop the quantum theory are suggested by the discussion above. In the initial Lagrangean we change coordinates

$$
x_{L}^{\mu}=x^{\mu}+i \theta_{i}^{a} \sigma_{a b}^{\mu} \bar{\theta}^{\dot{b} i}
$$

We can then write

$$
\omega^{\mu}=\dot{x}_{L}^{\mu}-2 i \dot{\theta}^{a}{ }_{i} \sigma_{a \dot{b}}^{\mu} \bar{\theta}^{\dot{b} i}
$$


The new momenta associated to the coordinates $\left(x_{I}^{\mu}, \theta^{a}, \bar{\theta}^{\dot{a}}\right)$ are

$$
\begin{gathered}
p_{\mu}^{L}=e^{-1} \omega_{\mu} \\
\pi_{a i}^{L}=-2 i p_{\mu}^{L} \sigma_{a \dot{a}}^{\mu} \bar{\theta}^{\dot{a}}{ }_{i} \\
\bar{\pi}_{\dot{a}}^{L}{ }^{i}=0 .
\end{gathered}
$$

We may also define

$$
\begin{aligned}
& d_{a i}^{L}= \pi_{a i}^{L}+2 i p_{\mu}^{L} \sigma_{a \dot{a}}^{\mu} \bar{\theta}^{\dot{a}}{ }_{i} \\
& \bar{d}_{\dot{a}}^{L}{ }^{i}=\bar{\pi}_{\dot{a}}^{L}{ }^{i}
\end{aligned}
$$

and we have,

$$
\left\{d_{a i}^{L}, \bar{d}_{\dot{a}}^{L}{ }^{j}\right\}=\delta_{i}{ }^{j} 2 i \sigma_{a \dot{a}}^{\mu} p_{\mu}^{L}=C_{a i \dot{a}}{ }^{j}
$$

The Dirac brackets are then given by

$$
\begin{gathered}
\left\{x_{L}^{\mu}, x_{L}^{\nu}\right\}_{\mathrm{D}}=\left\{x_{L}^{\mu}, \theta^{a i}\right\}_{\mathrm{D}}=\left\{\theta^{a i}, \theta^{b j}\right\}_{\mathrm{D}}=0 \\
\left\{x_{L}^{\mu}, p_{\nu}^{L}\right\}_{\mathrm{D}}=\delta_{\nu}^{\mu} \\
\left\{\pi_{a i}^{L}, \theta^{b j}\right\}_{\mathrm{D}}=-\delta_{i}^{j} \delta_{a}{ }^{b} .
\end{gathered}
$$

The resulting Hilbert space is the set of chiral superfields. The anti-chiral sector may be obtained along the same lines.

\section{The quantum algebra}

The chiral variables $X_{\mu}^{L}$ parameterize the physical degrees of freedom of the superparticle and give a solution for the classical dynamic problem, but to formulate the quantum theory of this system one has still to represent the Dirac algebra (17)-(21) in terms of a set of operators in terms of Heisenberg commutators following the rule $\{\cdot, \cdot\}_{\mathrm{D}} \rightarrow-i[\cdot, \cdot]$ or $-i\{\cdot, \cdot\}$. (We use $[\cdot, \cdot]$ for commutators and $\{\cdot, \cdot\}$ for anticommutators since no confusion can arise with the classical brackets.). The set of chiral superfields has been already identified as one Hilbert space where the quantization program may be pursued. As we discuss below this choice is not unique and when due care are given to some details in the algebra the space of anti-chiral and the space of superfields associated to the irreducible tensor multiplet may also be considered.

The other problem that has to be faced, as was first noted by Casalbuoni, is to deal with the fact that the coordinates $x^{\mu}$ cannot represented multiplicatively because they do not commute. Taking this into account, our task is to find a set of operators $\hat{X}^{\mu}, \hat{\Theta}^{a}$ and $\bar{\Theta}^{\dot{a}}$ that satisfy the following algebra to which we will refer as the quantum algebra of the superparticle, 


$$
\begin{gathered}
{\left[\hat{P}_{\mu}, \hat{\Theta}^{a}\right]=\left[\hat{P}_{\mu}, \hat{\bar{\Theta}}^{\dot{a}}\right]=\left\{\hat{\Theta}^{a}, \hat{\Theta}^{b}\right\}=\left\{\hat{\bar{\Theta}}^{\dot{a}}, \hat{\bar{\Theta}}^{b}\right\}=0} \\
{\left[\hat{X}^{\mu}, \hat{P}^{\nu}\right]=i \eta^{\mu \nu}} \\
\left\{\hat{\Theta}^{a}, \hat{\bar{\Theta}}^{\dot{a}}\right\}=\frac{-1}{2 \hat{P}^{2}} \hat{P}_{\mu} \bar{\sigma}^{\mu \dot{a} a} \\
{\left[\hat{X}^{\mu}, \hat{\Theta}^{a}\right]=\frac{i}{2 \hat{P}^{2}} \hat{P}_{\nu} \bar{\sigma}^{\nu} \dot{a} a \hat{\Theta}^{b} \sigma^{\mu}{ }_{b \dot{a}}} \\
{\left[\hat{X}^{\mu}, \hat{\bar{\Theta}}^{\dot{a}}\right]=\frac{i}{2 \hat{P}^{2}} \hat{P}_{\nu} \bar{\sigma}^{\nu a} \hat{\bar{\Theta}}^{\dot{b}} \sigma^{\mu}{ }_{a \dot{b}}} \\
{\left[\hat{X}^{\mu}, \hat{X}^{\nu}\right]=\frac{-i \hat{S}^{\mu \nu}}{\hat{P}^{2}} .}
\end{gathered}
$$

At the quantum level we may also define

$$
\hat{\Pi}_{a}=-i \hat{P}_{\mu} \sigma_{a \dot{a}}^{\mu} \hat{\bar{\Theta}}^{\dot{a}} \quad \hat{\bar{\Pi}}_{\dot{a}}=-i \hat{P}_{\mu} \sigma_{a \dot{a}}^{\mu} \hat{\Theta}^{a}
$$

and consequently all commutators involving this quantities could be obtained from this relations. The operator $\hat{S}^{\mu \nu}$ that appears on the right hand side of equation (39) is the internal angular momentum corresponding to the representation defined by our operators. That is we should have that

$$
\hat{J}^{\mu \nu}=\hat{X}^{\mu} \hat{P}^{\nu}-\hat{X}^{\mu} \hat{P}^{\nu}+\hat{S}^{\mu \nu}
$$

together with $\hat{Q}_{a}, \hat{\bar{Q}}_{\dot{a}}$ and $\hat{P}_{\mu}$ satisfy the super Poincarè algebra.

In the functional space of all the wave functions defined on the Salam and Strathdee superspace the super Poincarè algebra is represented by the operators,

$$
\begin{gathered}
P_{\mu}=-i \partial_{\mu} \\
\Pi_{a}=-i \partial_{a} \quad \bar{\Pi}_{\dot{a}}=-i \bar{\partial}_{\dot{a}} \\
J_{\mu \nu}=L_{\mu \nu}+S_{\mu \nu}=X_{\mu} P_{\nu}-X_{\mu} P_{\nu}-\frac{1}{4}\left(\Theta \sigma_{\mu \nu} \Pi+\bar{\Pi} \bar{\sigma}_{\mu \nu} \bar{\Theta}\right) \\
Q_{a}=\Pi_{a}-i P_{\mu} \sigma_{a \dot{a}}^{\mu} \bar{\Theta}^{\dot{a}} \\
\bar{Q}_{\dot{a}}=-\bar{\Pi}_{\dot{a}}+i P_{\mu} \sigma_{a \dot{a}}^{\mu} \Theta^{a} .
\end{gathered}
$$

The operators $X_{\mu}, \Theta^{a}$, and $\bar{\Theta}^{\dot{a}}$ act multiplicatively (see (A.14) $)$. This representation of the super Poincarè algebra is not irreducible. Even after imposing the mass shell condition $\left(P^{2}+m^{2}\right) \Psi=0$ the resulting representation is reducible. Indeed it holds the following theorem.([18, [19], 22])

\section{Theorem:}

Let $\Psi\left(x^{\mu}, \theta^{a}, \bar{\theta}^{\dot{a}}\right)$ be a superfield that satisfies

$$
\left(P^{2}+m^{2}\right) \Psi=0
$$


then it can be written in a unique way $(m \neq 0)$ as

$$
\begin{gathered}
\Psi=\Psi_{\mathrm{C}}+\Psi_{A}+\Psi_{\mathrm{T}} \\
\Psi_{\mathrm{C}}=\mathbb{P}_{\mathrm{C}}^{m} \Psi \quad \Psi_{A}=\mathbb{P}_{\mathrm{A}}^{m} \Psi \quad \Psi_{\mathrm{T}}=\mathbb{P}_{\mathrm{T}}^{m} \Psi
\end{gathered}
$$

where the projector operators are

$$
\mathbb{P}_{\mathrm{A}}^{m}=\frac{1}{16 m^{2}} D^{2} \bar{D}^{2} \quad \mathbb{P}_{\mathrm{C}}^{m}=\frac{1}{16 m^{2}} \bar{D}^{2} D^{2} \quad \mathbb{P}_{\mathrm{T}}^{m}=\frac{-1}{8 m^{2}} \bar{D}_{\dot{a}} D^{2} \bar{D}^{\dot{a}}
$$

The operators representing the super Poincarè algebra commute with any of this projectors, meaning each of the subspaces bear a representation of the group which correspond respectively to the chiral, anti-chiral and irreducible tensor multiplets. In these subspaces the corresponding generators are obtained in the form $\mathbb{P}_{G} J_{\mu \nu} \mathbb{P}_{G}$ and $\mathbb{P}_{G} P_{\mu} \mathbb{P}_{G}$ with $\mathbb{P}_{G}$ the corresponding operator.

The observation above led us to construct a representation of the quantum algebra of the superparticle by considering operators acting on the subspaces of the superfields space defined by the chiral (C), anti-chiral (A) or tensorial (T) projectors. The restricted operators are defined by defined by

$$
\begin{aligned}
X_{G}^{\mu} & \equiv \mathbb{P}_{G} X^{\mu} \mathbb{P}_{G} \\
\Theta_{G}^{a} & \equiv \mathbb{P}_{G} \Theta^{a} \mathbb{P}_{G} \\
\bar{\Theta}_{G}^{\dot{a}} & \equiv \mathbb{P}_{G} \bar{\Theta}^{\dot{a}} \mathbb{P}_{G}
\end{aligned}
$$

with $\mathbb{P}_{G}$ any one of the projectors,

$$
\mathbb{P}_{\mathrm{A}}=\frac{-1}{16 P^{2}} D^{2} \bar{D}^{2}, \quad \mathbb{P}_{\mathrm{C}}=\frac{-1}{16 P^{2}} \bar{D}^{2} D^{2}, \quad \mathbb{P}_{\mathrm{T}}=\frac{1}{8 P^{2}} \bar{D}_{\dot{a}} D^{2} \bar{D}^{\dot{a}}
$$

Note that the projectors have been taken out of the mass shell.

Below we show explicitly that each set of operators satisfy the quantum algebra (34. 391). In each of the subspaces the operator which we denote in generic form as $\hat{S}^{\mu \nu}$ at the right hand side of (39) results to be the projected internal angular momentum in the corresponding subspace. We introduce the notation, $S_{G}^{\mu \nu} \equiv \mathbb{P}_{G} S^{\mu \nu} \mathbb{P}_{G}$ for these operators.

We begin discussing the representation acting on the chiral superfields which after the discussion at the final of the previous section should correspond to the quantum theory of the superparticle defined by the classical action (2). We present the result in the form of the following theorem.

\section{Theorem}

The set of operators defined by $X_{\mathrm{C}}^{\mu}=\mathbb{P}_{\mathrm{C}} X^{\mu} \mathbb{P}_{\mathrm{C}}, \Theta_{\mathrm{C}}^{a}=\mathbb{P}_{\mathrm{C}} \Theta^{a} \mathbb{P}_{\mathrm{C}}$ and $\bar{\Theta}_{\mathrm{C}}^{\dot{a}}=\mathbb{P}_{\mathrm{C}} \bar{\Theta}^{\dot{a}} \mathbb{P}_{\mathrm{C}}$ satisfy the algebra 34(39). The operator $S_{\mathrm{C}}^{\mu \nu} \equiv \mathbb{P}_{\mathrm{C}} S^{\mu \nu} \mathbb{P}_{\mathrm{C}}$ can be written as

$$
S_{\mathrm{C}}^{\mu \nu}=-\frac{1}{4}\left(\Theta_{\mathrm{C}} \sigma^{\mu \nu} \Pi_{\mathrm{C}}+\bar{\Pi}_{\mathrm{C}} \bar{\sigma}^{\mu \nu} \bar{\Theta}_{\mathrm{C}}\right)=\varepsilon^{\mu \nu \rho \lambda} P_{\rho} \Theta_{\mathrm{C}}^{a} \sigma_{\lambda a \dot{b}} \bar{\Theta}_{\mathrm{C}}^{\dot{b}}
$$

\section{Proof}

To compute the anticommutator $\left\{\Theta_{\mathrm{C}}^{a}, \Theta_{\mathrm{C}}^{b}\right\}$, substitute $\Theta_{\mathrm{C}}^{a}$ by $\mathbb{P}_{\mathrm{C}} \Theta^{a} \mathbb{P}_{\mathrm{C}}$,

$$
\left\{\Theta_{\mathrm{C}}^{a}, \Theta_{\mathrm{C}}^{b}\right\}=\left(\mathbb{P}_{\mathrm{C}} \Theta^{a} \mathbb{P}_{\mathrm{C}}\right)\left(\mathbb{P}_{\mathrm{C}} \Theta^{b} \mathbb{P}_{\mathrm{C}}\right)+\left(\mathbb{P}_{\mathrm{C}} \Theta^{b} \mathbb{P}_{\mathrm{C}}\right)\left(\mathbb{P}_{\mathrm{C}} \Theta^{a} \mathbb{P}_{\mathrm{C}}\right)
$$


and use the fact that $\mathbb{P}_{\mathrm{C}}^{2}=\mathbb{P}_{\mathrm{C}}$. Then note that

$$
\mathbb{P}_{\mathrm{C}} \Theta^{a} \mathbb{P}_{\mathrm{C}} \Theta^{b} \mathbb{P}_{\mathrm{C}}=\mathbb{P}_{\mathrm{C}} \Theta^{a}\left[\mathbb{P}_{\mathrm{C}}, \Theta^{b}\right] \mathbb{P}_{\mathrm{C}}+\mathbb{P}_{\mathrm{C}} \Theta^{a} \Theta^{b} \mathbb{P}_{\mathrm{C}}=\mathbb{P}_{\mathrm{C}} \Theta^{a} \Theta^{b} \mathbb{P}_{\mathrm{C}}
$$

With the help of $\left(\right.$ A.23) observe that $\left[\mathbb{P}_{\mathrm{C}}, \Theta^{b}\right] \mathbb{P}_{\mathrm{C}}=0$. Then,

$$
\left\{\Theta_{\mathrm{C}}^{a}, \Theta_{\mathrm{C}}^{b}\right\}=\mathbb{P}_{\mathrm{C}}\left(\Theta^{a} \Theta^{b}+\Theta^{b} \Theta^{a}\right) \mathbb{P}_{\mathrm{C}}=0
$$

The anticommutator $\left\{\bar{\Theta}_{\mathrm{C}}^{\dot{a}}, \bar{\Theta}_{\mathrm{C}}^{\dot{b}}\right\}=0$ is also straightforward. To address the first non zero commutator:

$$
\left\{\Theta_{\mathrm{C}}^{a}, \bar{\Theta}_{\mathrm{C}}^{\dot{a}}\right\}=\mathbb{P}_{\mathrm{C}} \Theta^{a} \mathbb{P}_{\mathrm{C}} \bar{\Theta}^{\dot{a}} \mathbb{P}_{\mathrm{C}}+\mathbb{P}_{\mathrm{C}} \bar{\Theta}^{\dot{a}} \mathbb{P}_{\mathrm{C}} \Theta^{a} \mathbb{P}_{\mathrm{C}}
$$

use again the formula (A.23) to prove that

$$
\begin{gathered}
\left\{\Theta_{\mathrm{C}}^{a}, \bar{\Theta}_{\mathrm{C}}^{\dot{a}}\right\}=\mathbb{P}_{\mathrm{C}}\left[\Theta^{a}, \mathbb{P}_{\mathrm{C}}\right] \bar{\Theta}^{\dot{a}} \mathbb{P}_{\mathrm{C}}+\mathbb{P}_{\mathrm{C}} \bar{\Theta}^{\dot{a}}\left[\mathbb{P}_{\mathrm{C}}, \Theta^{a}\right] \mathbb{P}_{\mathrm{C}}= \\
\frac{-1}{8 P^{2}} \mathbb{P}_{\mathrm{C}}\left(-\bar{D}^{2} D^{a} \bar{\Theta}^{\dot{a}}+\bar{\Theta}^{\dot{a}} \bar{D}^{2} D^{a}\right) \mathbb{P}_{\mathrm{C}}= \\
\frac{-1}{8 P^{2}} \mathbb{P}_{\mathrm{C}}\left(-\left[\bar{D}^{2}, D^{a}\right] \bar{\Theta}^{\dot{a}}+\bar{\Theta}^{\dot{a}}\left[\bar{D}^{2}, D^{a}\right]\right) \mathbb{P}_{\mathrm{C}}= \\
\frac{-1}{8 P^{2}} \mathbb{P}_{\mathrm{C}}\left(4 i \bar{\sigma}^{\mu \dot{b} a} \bar{D}_{\dot{b}} \partial_{\mu} \bar{\Theta}^{\dot{a}}+\bar{\Theta}^{\dot{a}}\left(-4 i \bar{\sigma}^{\mu \dot{b} a} \bar{D}_{\dot{b}} \partial_{\mu}\right)\right) \mathbb{P}_{\mathrm{C}}= \\
\mathbb{P}_{\mathrm{C}}\left(\frac{4 i}{8 P^{2}} \bar{\sigma}^{\mu \dot{a} a} \partial_{\mu}\right) \mathbb{P}_{\mathrm{C}}=-\frac{1}{2 P^{2}} \bar{\sigma}^{\mu \dot{a} a} P_{\mu} .
\end{gathered}
$$

The next task is to compute $\left[X_{\mathrm{C}}^{\mu}, \Theta_{\mathrm{C}}^{a}\right]$. The tricks to be used are similar:

$$
\left[X_{\mathrm{C}}^{\mu}, \Theta_{\mathrm{C}}^{a}\right]=\mathbb{P}_{\mathrm{C}} X^{\mu} \mathbb{P}_{\mathrm{C}} \Theta^{a} \mathbb{P}_{\mathrm{C}}-\mathbb{P}_{\mathrm{C}} \Theta^{a} \mathbb{P}_{\mathrm{C}} X^{\mu} \mathbb{P}_{\mathrm{C}}=\mathbb{P}_{\mathrm{C}} X^{\mu}\left[\mathbb{P}_{\mathrm{C}}, \Theta^{a}\right] \mathbb{P}_{\mathrm{C}}-\mathbb{P}_{\mathrm{C}}\left[\Theta^{a}, \mathbb{P}_{\mathrm{C}}\right] X^{\mu} \mathbb{P}_{\mathrm{C}}
$$

Recalling once again that $\left[\mathbb{P}_{\mathrm{C}}, \Theta^{a}\right] \mathbb{P}_{\mathrm{C}}=0$,

$$
\begin{gathered}
{\left[X_{\mathrm{C}}^{\mu}, \Theta_{\mathrm{C}}^{a}\right]=\frac{-1}{8 P^{2}} \mathbb{P}_{\mathrm{C}} \bar{D}^{2} D^{a} X^{\mu} \mathbb{P}_{\mathrm{C}}=\frac{-1}{8 P^{2}} \mathbb{P}_{\mathrm{C}}\left[\bar{D}^{2}, D^{a}\right] X^{\mu} \mathbb{P}_{\mathrm{C}}=} \\
\frac{4 i}{8 P^{2}} \mathbb{P}_{\mathrm{C}} \bar{\sigma}^{\nu \dot{b} a} \partial_{\nu} \bar{D}_{\dot{b}} X^{\mu} \mathbb{P}_{\mathrm{C}}=\mathbb{P}_{\mathrm{C}}\left(\frac{i P_{\nu}}{P^{2}} \Theta^{b} \sigma_{b \dot{b}}^{\mu} \bar{\sigma}^{\nu} \dot{b} a\right) \mathbb{P}_{\mathrm{C}} .
\end{gathered}
$$

The next commutator is $\left[X_{\mathrm{C}}^{\mu}, X_{\mathrm{C}}^{\nu}\right]$ and is the most involved

$$
\begin{aligned}
& {\left[X_{\mathrm{C}}^{\mu}, X_{\mathrm{C}}^{\nu}\right]=\mathbb{P}_{\mathrm{C}} X^{\mu} \mathbb{P}_{\mathrm{C}} X^{\nu} \mathbb{P}_{\mathrm{C}}-\mathbb{P}_{\mathrm{C}} X^{\nu} \mathbb{P}_{\mathrm{C}} X^{\mu} \mathbb{P}_{\mathrm{C}}=} \\
& \quad=\mathbb{P}_{\mathrm{C}}\left[X^{\mu}, \mathbb{P}_{\mathrm{C}}\right] X^{\nu} \mathbb{P}_{\mathrm{C}}-\mathbb{P}_{\mathrm{C}}\left[X^{\nu}, \mathbb{P}_{\mathrm{C}}\right] X^{\mu} \mathbb{P}_{\mathrm{C}} .
\end{aligned}
$$

Using formulas (A.27, A.20) and (A.21), we can write

$$
\begin{gathered}
{\left[X^{\mu}, \mathbb{P}_{\mathrm{C}}\right]=-\frac{1}{P^{2}}\left[X^{\mu}, \bar{D}^{2} D^{2}\right]-\frac{1}{16}\left[X^{\mu}, \frac{1}{P^{2}}\right] \bar{D}^{2} D^{2}=} \\
=\frac{-1}{16 P^{2}}\left(\bar{D}^{2}\left[X^{\mu}, D^{2}\right]+\left[X^{\mu}, \bar{D}^{2}\right] D^{2}\right)-\frac{i}{8} \frac{P^{\mu}}{P^{2}} \frac{1}{P^{2}} \bar{D}^{2} D^{2}= \\
=\frac{i}{8}\left(\bar{D}^{2}\left(D \sigma^{\mu} \bar{\Theta}\right)-\left(\Theta \sigma^{\mu} \bar{D}\right) D^{2}\right)+\frac{2 i P^{\mu}}{P^{2}} \mathbb{P}_{\mathrm{C}} .
\end{gathered}
$$


With this computation the commutator is

$$
\begin{gathered}
{\left[X_{\mathrm{C}}^{\mu}, X_{\mathrm{C}}^{\nu}\right]=\frac{i}{8 P^{2}} \mathbb{P}_{\mathrm{C}}\left(\bar{D}^{2}\left(D \sigma^{\mu} \bar{\Theta}\right) X^{\nu}-\left(\Theta \sigma^{\mu} \bar{D}\right) D^{2} X^{\nu}+(\mu \leftrightarrow \nu)\right) \mathbb{P}_{\mathrm{C}}-} \\
-\frac{2 i}{P^{2}} \mathbb{P}_{\mathrm{C}}\left(X^{\mu} P^{\nu}-X^{\nu} P^{\mu}\right) \mathbb{P}_{\mathrm{C}}
\end{gathered}
$$

We recognize a term proportional to $L_{\mathrm{C}}^{\mu \nu}$. To further simplify the other term note that $\mathbb{P}_{\mathrm{C}} \bar{D}^{2} D^{a}=\mathbb{P}_{\mathrm{C}}\left[D^{2}, D^{a}\right]$ and $\mathbb{P}_{\mathrm{C}} \Theta^{a} \bar{D}^{\dot{a}} D^{2}=\mathbb{P}_{\mathrm{C}} \Theta^{a}\left[\bar{D}^{\dot{a}}, D^{2}\right]$. Using formulas (A.16) and (A.17) the commutator reads

$$
\begin{gathered}
{\left[X_{\mathrm{C}}^{\mu}, X_{\mathrm{C}}^{\nu}\right]=\frac{2 i}{P^{2}} L_{\mathrm{C}}^{\mu \nu}+} \\
+\frac{i(-4 i)}{8 P^{2}} \mathbb{P}_{\mathrm{C}}\left(\bar{D} \bar{\sigma}^{\lambda} \sigma^{\mu} \bar{\Theta} \partial_{\lambda} X^{\nu}-\Theta \sigma^{\mu} \bar{\sigma}^{\lambda} D \partial_{\lambda} X^{\nu}-(\mu \leftrightarrow \nu)\right) \mathbb{P}_{\mathrm{C}} \\
{\left[X_{\mathrm{C}}^{\mu}, X_{\mathrm{C}}^{\nu}\right]=\frac{2 i}{P^{2}} L_{\mathrm{C}}^{\mu \nu}+\frac{1}{2 P^{2}}\left(I_{1}^{\mu \nu}+I_{2}^{\mu \nu}\right)}
\end{gathered}
$$

To disentangle this equation first write $\partial_{\lambda} X^{\mu}=\delta_{\lambda}{ }^{\mu}+X^{\mu} \partial_{\lambda}$ so that

$$
\begin{gathered}
I_{1}^{\mu \nu}=\mathbb{P}_{\mathrm{C}}\left(\bar{D} \bar{\sigma}^{\lambda} \sigma^{\mu} \bar{\Theta} \partial_{\lambda} X^{\nu}-(\mu \leftrightarrow \nu)\right) \mathbb{P}_{\mathrm{C}}= \\
\mathbb{P}_{\mathrm{C}}\left(\bar{D} \bar{\sigma}^{\nu} \sigma^{\mu} \bar{\Theta}-\operatorname{Tr}\left(\bar{\sigma}^{\lambda} \sigma^{\mu}\right) X^{\nu} \partial_{\lambda}-\left(\bar{\sigma}^{\lambda} \sigma^{\mu}\right)^{\dot{a}}{ }_{\dot{b}} \bar{\Theta}^{\dot{b}}\left(\bar{D}_{\dot{a}} X^{\nu}\right) \partial_{\lambda}-(\mu \leftrightarrow \nu)\right) \mathbb{P}_{\mathrm{C}}
\end{gathered}
$$

The first of this terms is $0, \mathbb{P}_{\mathrm{C}} \bar{D} \bar{\sigma}^{\mu \nu} \bar{\Theta} \mathbb{P}_{\mathrm{C}}=0$ because $\operatorname{Tr}\left(\sigma^{\mu \nu}\right)=0$. The second is $2 \mathbb{P}_{\mathrm{C}}\left(X^{\nu} \partial^{\mu}-X^{\mu} \partial^{\nu}\right) \mathbb{P}_{\mathrm{C}}=-2 i L_{\mathrm{C}}^{\mu \nu}$. The last piece is

$$
\mathbb{P}_{\mathrm{C}}\left(i\left(\bar{\sigma}^{\lambda} \sigma^{\mu}\right)^{\dot{a}}{ }_{\dot{b}} \bar{\Theta}^{\dot{b}} \sigma_{b \dot{a}}^{\nu} \Theta^{a} \partial_{\lambda}-(\mu \leftrightarrow \nu)\right) \mathbb{P}_{\mathrm{C}}=\mathbb{P}_{\mathrm{C}}\left(\Theta\left(\sigma^{\nu} \bar{\sigma}^{\lambda} \sigma^{\mu}-\sigma^{\mu} \bar{\sigma}^{\lambda} \sigma^{\nu}\right) \bar{\Theta} P_{\lambda}\right) \mathbb{P}_{\mathrm{C}}
$$

Using the identity (A.5) $I_{1}^{\mu \nu}$ is given by

$$
I_{1}^{\mu \nu}=-2 i L_{\mathrm{C}}^{\mu \nu}-2 i \varepsilon^{\mu \nu \alpha \lambda} \mathbb{P}_{\mathrm{C}} \Theta \sigma_{\lambda} \bar{\Theta} \mathbb{P}_{\mathrm{C}}
$$

The other term, $I_{2}^{\mu \nu}$ is simpler

$$
\begin{gathered}
I_{2}^{\mu \nu}=-\mathbb{P}_{\mathrm{C}}\left(\Theta \sigma^{\mu} \bar{\sigma}^{\lambda} D \partial_{\lambda} X^{\nu}-(\mu \leftrightarrow \nu)\right) \mathbb{P}_{\mathrm{C}}= \\
=-\mathbb{P}_{\mathrm{C}}\left(\left(\sigma^{\mu} \bar{\sigma}^{\lambda}\right)_{a}{ }^{b}\left\{\Theta^{a}, D_{b}\right\} \partial_{\lambda} X^{\nu}-(\mu \leftrightarrow \nu)\right) \mathbb{P}_{\mathrm{C}}= \\
=2 \mathbb{P}_{\mathrm{C}}\left(\partial^{\mu} X^{\nu}-\partial^{\nu} X^{\mu}\right) \mathbb{P}_{\mathrm{C}}=-2 i L_{\mathrm{C}}^{\mu \nu}
\end{gathered}
$$

Taking all pieces together

$$
\left[X_{\mathrm{C}}^{\mu}, X_{\mathrm{C}}^{\nu}\right]=\frac{2 i}{P^{2}} L_{\mathrm{C}}^{\mu \nu}+\frac{1}{2 P^{2}}\left(-2 i L_{\mathrm{C}}^{\mu \nu}-2 i \varepsilon^{\mu \nu \alpha \lambda} \mathbb{P}_{\mathrm{C}} \Theta \sigma_{\lambda} \bar{\Theta} \mathbb{P}_{\mathrm{C}}-2 i L_{\mathrm{C}}^{\mu \nu}\right)
$$

Summing up we get,

$$
\left[X_{\mathrm{C}}^{\mu}, X_{\mathrm{C}}^{\nu}\right]=\frac{-i}{P^{2}} S_{\mathrm{C}}^{\mu \nu}
$$


In this case $S_{\mathrm{C}}^{\mu \nu}$ can be written in terms of the corresponding projected operators. To this end note that $\mathbb{P}_{\mathrm{C}} \bar{\Theta}^{\dot{a}} \Theta^{a} \mathbb{P}_{\mathrm{C}}=\mathbb{P}_{\mathrm{C}} \bar{\Theta}^{\dot{a}} \mathbb{P}_{\mathrm{C}} \Theta^{a} \mathbb{P}_{\mathrm{C}}$. since $\left[\Theta^{a}, \mathbb{P}_{\mathrm{C}}\right] \mathbb{P}_{\mathrm{C}}=0$. We have then,

$$
S_{\mathrm{C}}^{\mu \nu}=\mathbb{P}_{\mathrm{C}} S^{\mu \nu} \mathbb{P}_{\mathrm{C}}=\mathbb{P}_{\mathrm{C}} \varepsilon^{\mu \nu \rho \lambda} P_{\rho} \Theta^{a} \sigma_{\lambda a \dot{b}} \bar{\Theta}^{\dot{b}} \mathbb{P}_{\mathrm{C}}=\varepsilon^{\mu \nu \rho \lambda} P_{\rho} \Theta_{\mathrm{C}}^{a} \sigma_{\lambda a \dot{b}} \bar{\Theta}_{\mathrm{C}}^{\dot{b}}
$$

which satisfies Pryce's constraint $P_{\mu} S_{\mathrm{C}}^{\mu \nu}=0$

This end the proof of the theorem.

The commutators (60) 6272) realize the quantum algebra of the standard massive superparticle and hence we have a complete solution for the quantization of this system. The computations with the anti-chiral projector are completely analogous and led to exactly the same algebra. This give an equivalent but different covariant solution to the quantization of the superparticle.

We consider now the representation of the algebra using the tensorial projector.

\section{Theorem}

The set of operators defined by $X_{\mathrm{T}}^{\mu}=\mathbb{P}_{\mathrm{T}} X^{\mu} \mathbb{P}_{\mathrm{T}}, \Theta_{\mathrm{T}}^{a}=\mathbb{P}_{\mathrm{T}} \Theta^{a} \mathbb{P}_{\mathrm{T}}$ and $\bar{\Theta}_{\mathrm{T}}^{\dot{a}}=\mathbb{P}_{\mathrm{T}} \bar{\Theta}^{\dot{a}} \mathbb{P}_{\mathrm{T}}$ satisfy the algebra (34.39). The operator $S_{\mathrm{T}}^{\mu \nu} \equiv \mathbb{P}_{\mathrm{T}} S^{\mu \nu} \mathbb{P}_{\mathrm{T}}$ can be written as

$$
S_{\mathrm{T}}^{\mu \nu}=\tilde{S}_{\mathrm{T}}^{\mu \nu}+\frac{P_{\alpha}}{4 P^{2}} \varepsilon^{\mu \nu \alpha \lambda} \mathbb{P}_{\mathrm{T}} \bar{D} \bar{\sigma}_{\lambda} D \mathbb{P}_{\mathrm{T}}
$$

with

$$
\tilde{S}_{\mathrm{T}}^{\mu \nu}=-\frac{1}{4}\left(\Theta_{\mathrm{T}} \sigma^{\mu \nu} \Pi_{\mathrm{T}}+\bar{\Pi}_{\mathrm{T}} \bar{\sigma}^{\mu \nu} \bar{\Theta}_{\mathrm{T}}\right)
$$

\section{Proof}

The computation of $\left\{\Theta_{\mathrm{T}}^{a}, \Theta_{\mathrm{T}}^{b}\right\}=0$ and $\left\{\bar{\Theta}_{\mathrm{T}}^{\dot{a}}, \bar{\Theta}_{\mathrm{T}}^{\dot{b}}\right\}=0$ are again straightforward. Consider,

$$
\left\{\Theta_{\mathrm{T}}^{a}, \bar{\Theta}_{\mathrm{T}}^{\dot{a}}\right\}=\mathbb{P}_{\mathrm{T}} \Theta^{a} \mathbb{P}_{\mathrm{T}} \bar{\Theta}^{\dot{a}} \mathbb{P}_{\mathrm{T}}+\mathbb{P}_{\mathrm{T}} \bar{\Theta}^{\dot{a}} \mathbb{P}_{\mathrm{T}} \Theta^{a} \mathbb{P}_{\mathrm{T}}
$$

Taking advantage of (A.22) and $\bar{D}^{2} \mathbb{P}_{\mathrm{T}}=\mathbb{P}_{\mathrm{T}} \bar{D}^{2}=0$,

$$
\begin{gathered}
\left\{\Theta_{\mathrm{T}}^{a}, \bar{\Theta}_{\mathrm{T}}^{\dot{a}}\right\}=\mathbb{P}_{\mathrm{T}}\left[\Theta^{a}, \mathbb{P}_{\mathrm{T}}\right] \bar{\Theta}^{\dot{a}} \mathbb{P}_{\mathrm{T}}+\mathbb{P}_{\mathrm{T}} \bar{\Theta}^{\dot{a}}\left[\mathbb{P}_{\mathrm{T}}, \Theta^{a}\right] \mathbb{P}_{\mathrm{T}}= \\
\frac{1}{8 P^{2}} \mathbb{P}_{\mathrm{T}}\left(-\left\{D^{a}, \bar{D}^{2}\right\} \bar{\Theta}^{\dot{a}}+\bar{\Theta}^{\dot{a}}\left\{\bar{D}^{2}, D^{a}\right\}\right) \mathbb{P}_{\mathrm{T}}= \\
\frac{1}{8 P^{2}} \mathbb{P}_{\mathrm{T}}\left(-\left[D^{a}, \bar{D}^{2}\right] \bar{\Theta}^{\dot{a}}+\bar{\Theta}^{\dot{a}}\left[\bar{D}^{2}, D^{a}\right]\right) \mathbb{P}_{\mathrm{T}} .
\end{gathered}
$$

Recalling again (A.16),

$$
\left\{\Theta_{\mathrm{T}}^{a}, \bar{\Theta}_{\mathrm{T}}^{\dot{a}}\right\}=\frac{-4 i \bar{\sigma}^{\mu \dot{b} a}}{8 P^{2}} \mathbb{P}_{\mathrm{T}}\left(\bar{D}_{\dot{b}} \bar{\Theta}^{\dot{a}}+\bar{\Theta}^{\dot{a}} \bar{D}_{\dot{b}}\right) \mathbb{P}_{\mathrm{T}} \partial_{\mu}=\mathbb{P}_{\mathrm{T}}\left(\frac{4 i}{8 P^{2}} \bar{\sigma}^{\mu \dot{a} a} \partial_{\mu}\right) \mathbb{P}_{\mathrm{T}}
$$

Next compute,

$$
\begin{gathered}
{\left[X_{\mathrm{T}}^{\mu}, \Theta_{\mathrm{T}}^{a}\right]=\mathbb{P}_{\mathrm{T}} X^{\mu} \mathbb{P}_{\mathrm{T}} \Theta^{a} \mathbb{P}_{\mathrm{T}}-\mathbb{P}_{\mathrm{T}} \Theta^{a} \mathbb{P}_{\mathrm{T}} X^{\mu} \mathbb{P}_{\mathrm{T}}=\mathbb{P}_{\mathrm{T}} X^{\mu}\left[\mathbb{P}_{\mathrm{T}}, \Theta^{a}\right] \mathbb{P}_{\mathrm{T}}-\mathbb{P}_{\mathrm{T}}\left[\Theta^{a}, \mathbb{P}_{\mathrm{T}}\right] X^{\mu} \mathbb{P}_{\mathrm{T}}=} \\
\frac{1}{8 P^{2}} \mathbb{P}_{\mathrm{T}}\left(X^{\mu}\left[\bar{D}^{2}, D^{a}\right]+\left[D^{a}, \bar{D}^{2}\right] X^{\mu}\right) \mathbb{P}_{\mathrm{T}}= \\
\frac{1}{8 P^{2}} \mathbb{P}_{\mathrm{T}}\left(-X^{\mu} 4 i \bar{\sigma}^{\nu} \dot{b} a \bar{D}_{\dot{b}} \partial_{\nu}+4 i \bar{\sigma}^{\nu} \dot{b} a \bar{D}_{\dot{b}} \partial_{\nu} X^{\mu}\right) \mathbb{P}_{\mathrm{T}}= \\
\frac{\bar{\sigma}^{\nu} \dot{b} a}{2 i P^{2}} \mathbb{P}_{\mathrm{T}}\left(X^{\mu} \bar{D}_{\dot{b}}-\bar{D}_{\dot{b}} X^{\mu}\right) \mathbb{P}_{\mathrm{T}} \partial_{\nu}=\mathbb{P}_{\mathrm{T}}\left(\frac{i}{2 P^{2}} \Theta^{b} \sigma^{\mu}{ }_{b \dot{b}} \bar{\sigma}^{\nu} \dot{b} a\right) \mathbb{P}_{\mathrm{T}} P_{\nu}
\end{gathered}
$$


Finally consider,

$$
\left[X_{\mathrm{T}}^{\mu}, X_{\mathrm{T}}^{\nu}\right]=\mathbb{P}_{\mathrm{T}} X^{\mu} \mathbb{P}_{\mathrm{T}} X^{\nu} \mathbb{P}_{\mathrm{T}}-\mathbb{P}_{\mathrm{T}} X^{\nu} \mathbb{P}_{\mathrm{T}} X^{\mu} \mathbb{P}_{\mathrm{T}}=\mathbb{P}_{\mathrm{T}}\left[X^{\mu}, \mathbb{P}_{\mathrm{T}}\right] X^{\nu} \mathbb{P}_{\mathrm{T}}-\mathbb{P}_{\mathrm{T}}\left[X^{\nu}, \mathbb{P}_{\mathrm{T}}\right] X^{\mu} \mathbb{P}_{\mathrm{T}}
$$

Taking into account the previous computation

$$
\begin{gathered}
\mathbb{P}_{\mathrm{T}}\left[X^{\mu}, \mathbb{P}_{\mathrm{T}}\right]=-\mathbb{P}_{\mathrm{T}}\left[X^{\mu}, \mathbb{P}_{\mathrm{C}}\right]-\mathbb{P}_{\mathrm{T}}\left[X^{\mu}, \mathbb{P}_{\mathrm{A}}\right]= \\
\frac{-1}{16 P^{2}} \mathbb{P}_{\mathrm{T}}\left(\bar{D}^{2}\left[D^{2}, X^{\mu}\right]+\left[\bar{D}^{2}, X^{\mu}\right] D^{2}+D^{2}\left[\bar{D}^{2}, X^{\mu}\right]+\left[D^{2}, X^{\mu}\right] \bar{D}^{2}\right)= \\
\frac{-1}{16 P^{2}} \mathbb{P}_{\mathrm{T}}\left(\left[\bar{D}^{2}, X^{\mu}\right] D^{2}+\left[D^{2}, X^{\mu}\right] \bar{D}^{2}\right)
\end{gathered}
$$

Introducing this value in the commutator and using (A.20) and (A.21)

$$
\begin{gathered}
{\left[X_{\mathrm{T}}^{\mu}, X_{\mathrm{T}}^{\nu}\right]=\frac{2 i}{16 P^{2}} \mathbb{P}_{\mathrm{T}}\left(D \sigma^{\mu} \bar{\Theta} \bar{D}^{2}-\Theta \sigma^{\mu} \bar{D} D^{2}\right) X^{\nu} \mathbb{P}_{\mathrm{T}}-(\mu \leftrightarrow \nu)=} \\
\frac{i}{8 P^{2}} \mathbb{P}_{\mathrm{T}}\left(D \sigma^{\mu} \bar{\Theta}\left[\bar{D}^{2}, X^{\nu}\right]-\Theta \sigma^{\mu} \bar{D}\left[D^{2}, X^{\nu}\right]\right) \mathbb{P}_{\mathrm{T}}-(\mu \leftrightarrow \nu)= \\
\frac{1}{4 P^{2}} \mathbb{P}_{\mathrm{T}}\left(D \sigma^{\mu} \bar{\Theta} \Theta \sigma^{\nu} \bar{D}+\Theta \sigma^{\mu} \bar{D} D \sigma^{\nu} \bar{\Theta}\right) \mathbb{P}_{\mathrm{T}}-(\mu \leftrightarrow \nu)
\end{gathered}
$$

The last two terms in (3) can be written as

$$
\frac{1}{4 P^{2}} \mathbb{P}_{\mathrm{T}}\left(\left[D \sigma^{\nu} \bar{\Theta}, \Theta \sigma^{\nu} \bar{D}\right]-(\mu \leftrightarrow \nu)\right) \mathbb{P}_{\mathrm{T}}
$$

The commutator here is given by

$$
\begin{gathered}
{\left[D^{a} \sigma_{a \dot{a}}^{\nu} \bar{\Theta}^{\dot{a}}, \Theta^{b} \sigma^{\mu}{ }_{b \dot{b}} \bar{D}^{\dot{b}}\right]-(\mu \leftrightarrow \nu)=\sigma^{\nu}{ }_{a \dot{a}} \sigma^{\mu}{ }_{b \dot{b}}\left[D^{a} \bar{\Theta}^{\dot{a}}, \Theta^{b} \bar{D}^{\dot{b}}\right]-(\mu \leftrightarrow \nu)=} \\
\sigma^{\nu}{ }_{a \dot{a}} \sigma^{\mu}{ }_{b \dot{b}}\left(D^{a}\left[\bar{\Theta}^{\dot{a}}, \Theta^{b} \bar{D}^{\dot{b}}\right]+\left[D^{a}, \Theta^{b} \bar{D}^{\dot{b}}\right] \bar{\Theta}^{\dot{a}}\right)-(\mu \leftrightarrow \nu)= \\
\sigma^{\nu}{ }_{a \dot{a}} \sigma^{\mu}{ }_{b \dot{b}}\left(-D^{a} \Theta^{b}\left\{\bar{\Theta}^{\dot{a}}, \bar{D}^{\dot{b}}\right\}+\left\{D^{a}, \Theta^{b}\right\} \bar{D}^{\dot{b}} \bar{\Theta}^{\dot{a}}-\Theta^{b}\left\{D^{a}, \bar{D}^{\dot{b}}\right\} \bar{\Theta}^{\dot{a}}\right)-(\mu \leftrightarrow \nu)= \\
\sigma^{\nu}{ }_{a \dot{a}} \sigma^{\mu}{ }_{b \dot{b}}\left(-D^{a} \Theta^{b} \varepsilon^{\dot{a} \dot{b}}+\varepsilon^{a b} \bar{D}^{\dot{b}} \bar{\Theta}^{\dot{a}}-2 P_{\alpha} \bar{\sigma}^{\alpha \dot{b} a} \Theta^{b} \bar{\Theta}^{\dot{a}}\right)-(\mu \leftrightarrow \nu)
\end{gathered}
$$

The first term is

$$
\begin{aligned}
\sigma_{a \dot{a}}^{\nu} \sigma_{b \dot{b}}^{\mu}\left(-D^{a} \Theta^{b} \varepsilon^{\dot{a} \dot{b}}\right)- & (\mu \leftrightarrow \nu)=-\varepsilon^{\dot{a} \dot{b}}{\sigma^{\nu}}_{a \dot{a}} \sigma_{b \dot{b}}^{\mu} \varepsilon^{a c} D_{c} \Theta^{b}-(\mu \leftrightarrow \nu)= \\
& -\sigma^{\mu \nu}{ }_{b}{ }_{b} D_{c} \Theta^{b}=\Theta \sigma^{\mu \nu} D
\end{aligned}
$$

using that the matrix $\sigma^{\mu \nu}$ is traceless. A similar computation for the second term in equation (84) yields the result $-\bar{D} \bar{\sigma}^{\mu \nu} \bar{\Theta}$. With the aid of (A.5) the third and last term sum up to,

$$
-2 P_{\alpha} \Theta\left(\sigma^{\nu} \bar{\sigma}^{\alpha} \sigma^{\mu}-\sigma^{\mu} \bar{\sigma}^{\alpha} \sigma^{\nu}\right) \bar{\Theta}=4 i P_{\alpha} \varepsilon^{\nu \alpha \mu \lambda} \Theta \sigma_{\lambda} \bar{\Theta}
$$


and taking all the terms together ,

$$
\left[X_{\mathrm{T}}^{\mu}, X_{\mathrm{T}}^{\nu}\right]=\frac{1}{4 P^{2}} \mathbb{P}_{\mathrm{T}}\left(\Theta \sigma^{\mu \nu} D-\bar{D} \bar{\sigma}^{\mu \nu} \bar{\Theta}+4 i P_{\alpha} \varepsilon^{\nu \alpha \mu \lambda} \Theta \sigma_{\lambda} \bar{\Theta}\right) \mathbb{P}_{\mathrm{T}}
$$

Using the explicit form of $D_{a}$ and $\bar{D}_{\dot{a}}$ shown in the appendix and formula (A.4),

$$
\begin{gathered}
\frac{1}{4 P^{2}}\left(\Theta \sigma^{\mu \nu} D-\bar{D} \bar{\sigma}^{\mu \nu} \bar{\Theta}+4 i P_{\alpha} \varepsilon^{\nu \alpha \mu \lambda} \Theta \sigma_{\lambda} \bar{\Theta}\right)= \\
\frac{i}{4 P^{2}}\left(\Theta \sigma^{\mu \nu} \Pi+\bar{\Pi} \sigma^{\mu \nu} \bar{\Theta}\right)+\frac{1}{4 P^{2}}\left(-P_{\alpha} \Theta\left(\sigma^{\mu \nu} \sigma^{\alpha}+\sigma^{\alpha} \sigma^{\mu \nu}\right) \bar{\Theta}+4 i P_{\alpha} \varepsilon^{\nu \alpha \mu \lambda} \Theta \sigma_{\lambda} \bar{\Theta}\right)= \\
\frac{-i S^{\mu \nu}}{P^{2}}+\frac{P_{\alpha}}{4 P^{2}}\left(-4 i \varepsilon^{\mu \nu \alpha \lambda}+4 i \varepsilon^{\nu \alpha \mu \lambda}\right) \Theta \sigma_{\lambda} \bar{\Theta}=\frac{-i S^{\mu \nu}}{P^{2}}
\end{gathered}
$$

Finally,

$$
\left[X_{\mathrm{T}}^{\mu}, X_{\mathrm{T}}^{\nu}\right]=\frac{-i}{P^{2}} S_{\mathrm{T}}^{\mu \nu}
$$

with $S_{\mathrm{T}}^{\mu \nu}=\mathbb{P}_{\mathrm{T}} S^{\mu \nu} \mathbb{P}_{\mathrm{T}}$. Note that this is not equal to $(75)$ and therefore this set of operators do not correspond to the quantization of (2). The operator $S_{\mathrm{T}}^{\mu \nu}$ is not written solely in terms of the $T$ operators. There is an extra term that accounts for the internal super spin. We complete this observation with the following lemma.

\section{Lemma}

$$
S_{\mathrm{T}}^{\mu \nu}=\tilde{S}_{\mathrm{T}}^{\mu \nu}+\frac{P_{\alpha}}{4 P^{2}} \varepsilon^{\mu \nu \alpha \lambda} \mathbb{P}_{\mathrm{T}} \bar{D} \bar{\sigma}_{\lambda} D \mathbb{P}_{\mathrm{T}}
$$

Note also that in the chiral (or anti-chiral) case,

$$
P_{\alpha} \varepsilon^{\mu \nu \alpha \lambda} \mathbb{P}_{\mathrm{C}} \bar{D} \bar{\sigma}_{\lambda} D \mathbb{P}_{\mathrm{C}}=0
$$

\section{Proof}

To start insert $I=\mathbb{P}_{\mathrm{T}}+\mathbb{P}_{\mathrm{C}}+\mathbb{P}_{\mathrm{A}}$ in the formula for $S_{\mathrm{T}}^{\mu \nu}$

$$
\begin{aligned}
S_{\mathrm{T}}^{\mu \nu}= & \frac{-1}{4} \mathbb{P}_{\mathrm{T}}\left(\Theta \sigma^{\mu \nu}\left(\mathbb{P}_{\mathrm{T}}+\mathbb{P}_{\mathrm{C}}+\mathbb{P}_{\mathrm{A}}\right) \Pi+\bar{\Pi} \bar{\sigma}^{\mu \nu}\left(\mathbb{P}_{\mathrm{T}}+\mathbb{P}_{\mathrm{C}}+\mathbb{P}_{\mathrm{A}}\right) \bar{\Theta}\right) \mathbb{P}_{\mathrm{T}}= \\
& =\tilde{S}_{\mathrm{T}}^{\mu \nu}+\frac{-1}{4} \mathbb{P}_{\mathrm{T}}\left(\Theta \sigma^{\mu \nu}\left(\mathbb{P}_{\mathrm{C}}+\mathbb{P}_{\mathrm{A}}\right) \Pi+\bar{\Pi} \bar{\sigma}^{\mu \nu}\left(\mathbb{P}_{\mathrm{C}}+\mathbb{P}_{\mathrm{A}}\right) \bar{\Theta}\right) \mathbb{P}_{\mathrm{T}}
\end{aligned}
$$

Note that due to (A.23) the terms with $\mathbb{P}_{\mathrm{C}}$ disappear. Then with the aid of,

$$
\begin{gathered}
\Delta S_{\mathrm{T}}^{\mu \nu}=S_{\mathrm{T}}^{\mu \nu}-\tilde{S}_{\mathrm{T}}^{\mu \nu}=\frac{-1}{4} \mathbb{P}_{\mathrm{T}}\left(\left[\Theta^{a}, \mathbb{P}_{\mathrm{A}}\right] \Pi_{b} \sigma_{a}^{\mu \nu}{ }_{a}^{b}+\bar{\sigma}_{\dot{b}}^{\mu \nu}{ }_{\dot{a}} \bar{\Pi}_{\dot{a}}\left[\mathbb{P}_{\mathrm{A}}, \bar{\Theta}^{\dot{b}}\right]\right) \mathbb{P}_{\mathrm{T}}= \\
=\frac{-1}{4} \mathbb{P}_{\mathrm{T}}\left(\frac{1}{8 P^{2}} D^{a} \bar{D}^{2} \Pi_{b} \sigma_{a}^{\mu \nu}{ }_{a}{ }^{\prime}-\frac{1}{8 P^{2}} \bar{\sigma}_{\dot{b}}^{\mu \nu \dot{a}} \bar{\Pi}_{\dot{a}} D^{2} \bar{D}^{\dot{b}}\right) \mathbb{P}_{\mathrm{T}}= \\
=\frac{-i P_{\alpha}}{16 P^{2}} \mathbb{P}_{\mathrm{T}}\left[D\left(\sigma^{\mu \nu} \sigma^{\alpha}+\sigma^{\alpha} \bar{\sigma}^{\mu \nu}\right) \bar{D}\right] \mathbb{P}_{\mathrm{T}}= \\
=\frac{-P_{\alpha}}{4 P^{2}} \varepsilon^{\mu \nu \alpha \lambda} \mathbb{P}_{\mathrm{T}} D \sigma_{\lambda} \bar{D} \mathbb{P}_{\mathrm{T}}
\end{gathered}
$$


This last formula completes the proof of the lemma and the theorem.

The subspaces defined by the chiral and the tensor projectors bear irreducible representations of $N=1$ super Poincarè with superspin 0 and $1 / 2$ respectively. As final observation before closing this section let us compute the relevant Casimir operator for each of the representations we have obtained. We first define the analog of the Pauli-Lubanski four vector

$$
W_{\mu}=\frac{1}{2} \varepsilon_{\mu \nu \alpha \beta} \hat{P}^{\nu} \hat{J}^{\alpha \beta}+\frac{1}{8} \hat{\bar{Q}} \bar{\sigma}_{\mu} \hat{Q}
$$

where $\hat{P}_{\mu}, \hat{J}^{\mu \nu}, \hat{Q}_{a}$ and $\hat{\bar{Q}}_{\dot{a}}$ are the projected operators. This definition is taken from Salam and Strathdee [1] or Sokatchev 24. As we shall show below it is transverse in the spaces defined by the chiral and the tensor projectors, although it is not transverse in the general setting (readers may wish to compare this definition with that of [18] which is transverse from the beginning). With this four vector we construct a Casimir:

$$
W=W_{\mu} W^{\mu}
$$

In a given irreducible representation $W$ is equal to $-P^{2} Y(Y+1)$ with $Y$ is the super spin of the representation. For the chiral (and the anti-chiral of course) it is not difficult to show that

$$
W_{\mu}^{\mathrm{C}}=0
$$

Which implies naturally that $W_{\mathrm{C}}=0$ as we might expect.

For the quantum algebra defined by means of the tensorial projector, the non zero term came from the new term in the expression for the internal angular momentum

$$
\begin{gathered}
W_{\mu}^{\mathrm{T}}=\frac{-1}{4} \mathbb{P}_{\mathrm{T}} \bar{D} \bar{\sigma}_{\mu} D \mathbb{P}_{\mathrm{T}}-\frac{1}{2} P_{\mu} \mathbb{P}_{\mathrm{T}} \\
W_{\mathrm{T}}=\left(\frac{-1}{4} \mathbb{P}_{\mathrm{T}} \bar{D} \bar{\sigma}_{\mu} D \mathbb{P}_{\mathrm{T}}-\frac{1}{2} P_{\mu} \mathbb{P}_{\mathrm{T}}\right)\left(\frac{-1}{4} \mathbb{P}_{\mathrm{T}} \bar{D} \bar{\sigma}^{\mu} D \mathbb{P}_{\mathrm{T}}-\frac{1}{2} P^{\mu} \mathbb{P}_{\mathrm{T}}\right)= \\
=\mathbb{P}_{\mathrm{T}}\left(\frac{1}{16}\left(\bar{D} \bar{\sigma}_{\mu} D\right)\left(\bar{D} \bar{\sigma}^{\mu} D\right)+\frac{1}{8} P_{\mu} \bar{D} \bar{\sigma}^{\mu} D\right) \mathbb{P}_{\mathrm{T}}
\end{gathered}
$$

Now use (A.7) and Okubo's formula (A.26) to finally obtain,

$$
W_{\mathrm{T}}=-P^{2} \frac{1}{2}\left(\frac{1}{2}+1\right)
$$

\section{The superspin 1/2 superparticle}

In the previous section we show that the quantum algebra of the superspace may be realized by projecting the observables on the three supersymmetric sectors of the Hilbert space of superfields. The chiral and anti-chiral realizations correspond to the usual $D=4$ massive superparticle as we demonstrate in section 2 using the corresponding chiral or anti-chiral variables. We have learned that there are three kinds of superparticles in the 
Salam-Strathdee superspace. It is natural to ask which is the classical action which upon quantization give rise to the superspin $1 / 2$ superparticle. This action is given by

$$
S=\frac{1}{2} \int\left\{e^{-1} \omega^{\mu} \omega_{\mu}-e m^{2}+\ell\left(\dot{\theta}^{2}+\dot{\bar{\theta}}^{2}\right)\right\} d \tau
$$

The field $\ell(\tau)$ is a new variable that enters in the action besides the coordinates $\left(x^{\mu}, \theta^{a}, \bar{\theta}^{\dot{a}}\right)$. This system has no second class constraints. The condition $\pi_{\ell}=0$ is a first class constraint. Preservation of this constraint implies

$$
\dot{\theta}^{2}+\dot{\bar{\theta}}^{2}=0
$$

We also have,

$$
\pi_{a}+i p_{\mu} \sigma_{a \dot{b}}^{\mu} \dot{\bar{\theta}}^{\dot{b}}+\varepsilon_{a b} \ell \dot{\theta}^{b}=0
$$

and defining

$$
d_{a}=\pi_{a}+i p_{\mu} \sigma_{a \dot{b}}^{\mu} \dot{\bar{\Theta}}^{\dot{b}}=\varepsilon_{a b} \ell \dot{\theta}^{b}
$$

we end up for $\ell \neq 0$ with the following first class constraints

$$
\begin{gathered}
\pi_{\ell}=0 \\
p^{2}+m^{2}=0 \\
d^{2}+\bar{d}^{2}=0 .
\end{gathered}
$$

The quantum mechanics of this system is now straightforward. Imposing the constraints on a superfield $\Psi\left(x^{\mu}, \theta^{a}, \bar{\theta}^{\dot{a}}\right)$ we have

$$
\begin{aligned}
& \left(P^{2}+m^{2}\right) \Psi\left(x^{\mu}, \theta^{a}, \bar{\theta}^{\dot{a}}\right)=0 \\
& \left(D^{2}+\bar{D}^{2}\right) \Psi\left(x^{\mu}, \theta^{a}, \bar{\theta}^{\dot{a}}\right)=0
\end{aligned}
$$

which after simple algebraic manipulations led to

$$
\begin{aligned}
& \left(P^{2}+m^{2}\right) \Psi=0 \\
& D^{2} \Psi=\bar{D}^{2} \Psi=0
\end{aligned}
$$

In turn this equations are equivalent to

$$
\left(P^{2}+m^{2}\right) \Psi=0 \quad \mathbb{P}_{\mathrm{T}} \Psi=\Psi
$$

To prove this last statements observe that if (107) and (108) hold then $D^{2} \bar{D}^{2} \Psi=0$ and $\bar{D}^{2} D^{2} \Psi=0$ which imply that $\left\{D^{2}, \bar{D}^{2}\right\} \Psi=0$. With the identity (A.19) we now see that (111) holds which clearly imply that $D^{2} \Psi=\bar{D}^{2} \Psi=0$.

This establishes that the wave functions for this system are superfields restricted by the tensorial projector. We note that in our formulation the Weyl spinors satisfy a Klein Gordon field equation. This is equivalent to a Dirac equation for the uniquely associated Dirac spinor. 
Since this is a gauge system we need not to realize the complete algebra of the observables to extract the physical content. Nevertheless we observe that it should exist a covariant gauge fixing condition for which the algebra of eqs. (78), (179) and (89) for the quantum phase space variables is realized.

Some comments are in order. Action (100) with $\ell$ proportional to the inverse of the einbein $e(\tau)$ was first proposed by Volkov and Pashnev in [20] and was also considered in 3 . The resulting spectrum of physical states consists of the chiral , anti-chiral and irreducible tensor multiplets with related masses. Upon quantization the VolkovPashnev system leads to unitarity problems. This can be understood in the following way. If we keep $\ell$ fixed then there is only one first class constraint and the space of states is the whole space of wave functions. We can prove that in this space there is no supersymmetric positive definite inner product. It is necessary (in order to have a real representation of supersymmetry) that

$$
\left(Q_{a}\right)^{\dagger}=\bar{Q}_{\dot{a}} \quad\left(J_{\mu \nu}\right)^{\dagger}=J_{\mu \nu}
$$

This implies that

$$
\left(\Theta^{a}\right)^{\dagger}=\bar{\Theta}^{\dot{a}} \quad\left(\Pi_{a}\right)^{\dagger}=\bar{\Pi}_{\dot{a}}
$$

which in turn implies

$$
\left(D_{a}\right)^{\dagger}=-\bar{D}_{\dot{a}}
$$

Now consider a chiral superfield $\Psi\left(x^{\mu}, \theta^{a}, \bar{\theta}^{\dot{a}}\right)$ and let us assume that there is a scalar product such that $(\Psi, \Psi)=1$. We built the wave function $D_{a} \Psi$. It is easily seen with the use of A.29 that this wave function is in the space restricted by the tensorial projector. It satisfies,

$$
\left(D_{a} \Psi, D_{a} \Psi\right)=-\left(\Psi, \bar{D}_{\dot{a}} D_{a} \Psi\right)=-2 \sigma_{a \dot{a}}^{\mu}\left(\Psi, P_{\mu} \Psi\right)<0
$$

The last inequality follows from supersymmetry

$$
0<\left(Q_{a} \Psi, Q_{a} \Psi\right)=2 \sigma_{a \dot{a}}^{\mu}\left(\Psi, P_{\mu} \Psi\right)
$$

This is also the conclusion obtained by Volkov and Pashnev. The problem will always appear if one retains the whole space of superfields. In distinction, for our system $\ell(\tau)$ acts as an independent lagrange multiplier and we are restricted to the sector of the superfields space associated to the irreducible tensor multiplet which is a Hilbert space. In this case $\Psi$ and $Q_{a} \Psi$ cannot be simultaneously in the chosen Hilbert space. To sum up, it is possible to have a Hilbert space structure on the spaces projected by the chiral, anti-chiral or tensorial projectors but not in the whole space of superfields. For a related discussion see 21.

\section{$5 \quad$ Projectors and second class constraints}

In this section we put in a general setup our experience with the quantum algebra of the superparticle. Geometrically second class constraints restrict the symplectic manifold of 
the phase space to a symplectic submanifold and first class constraints further reduce it to a foliated Poisson manifold. When second class constraints are present, Dirac algorithm has to be handle with care. In the transition to quantum theory it may appear ordering problems and locality problems related with the inverse of the matrix of the constraints as an operator. At the classical level Darboux theorem assures that there exists a set of canonical coordinates but even if this set of coordinates is obtained it should be considered that the quantization may be not straightforward. It is useful and interesting to represent directly the Dirac algebra without dwelling in a specific choice of independent coordinates.

Let us consider the general case of a classical system with coordinates and momenta $\left(q^{i}, p_{j}\right)$ subject to second class constraints $\phi_{\alpha}\left(q^{i}, p_{j}\right)$ satisfying the algebra

$$
\left\{\phi_{\alpha}, \phi_{\beta}\right\}=C_{\alpha \beta}
$$

with the Dirac brackets given by

$$
\{F, G\}_{\mathrm{D}}=\{F, G\}-\left\{F, \phi_{\alpha}\right\} C^{\alpha \beta}\left\{\phi_{\beta}, G\right\} .
$$

First we note that since second class constraints reduce the phase space in quantum mechanics this should mean that the physical Hilbert space is a subspace of the whole Hilbert space $\mathcal{H}$. Any such subspace is characterized by a projection operator $\mathbb{P}$ with the physical Hilbert subspace given by $\mathbb{P} \mathcal{H}$. The operators acting on the physical space are those projected from the whole Hilbert space $\mathcal{H}$.

What we observe is that there should exist a projection operator $\mathbb{P}_{G}$ such that the quantum algebra is represented on the Hilbert subspace $G$ in a generalized Schrödinger picture by

$$
\begin{gathered}
Q_{G}^{i} \rightarrow \mathbb{P}_{G} Q^{i} \mathbb{P}_{G} \\
P_{G i} \rightarrow \mathbb{P}_{G}\left(-i \hbar \partial_{i}\right) \mathbb{P}_{G}
\end{gathered}
$$

As we saw in the case of the superparticle this proposal provides us with an elegant solution to the representation of the superparticle algebra.

\section{Conclusions and outlook}

In this paper we have presented the quantization of the massive superparticle from new points of view which reveal some aspects of the structure of the superspace previously unnoted. We have presented the canonical reduction of the classical action (2) to its physical degrees of freedom. This procedure identifies in the most direct way the wave functions of this system as the chiral superfields. We then have constructed an explicit representation of the quantum observables of the system acting on the space of chiral superfields. This have been achieved by considering the projection of the operators acting on arbitrary superfields on the set of chiral superfields. This procedure unravel also the possibility of having a different but related quantum algebra realized on the space of superfields associated to the irreducible tensor multiplet. To complete the physical 
picture we display a new classical action whose wave functions are shown to be precisely these superfields.

Let us turn now to possible generalizations of our results to other superparticle models. Increasing $N$ in $D=4$ does not represent a major complication for our method. For every $N$ there exist projection operators [22]. These could be used to construct the quantum algebra which should then be compared with the one arising from the quantization of higher $N$ superparticles. More complicated appears to be the generalization to higher dimensions where adequate projectors should be constructed.

In 1946 Snyder [25] noted that by introducing a fundamental length (call it $a$ ) in physics the commutator of two space-time operators might not vanish. He indeed proposed the formula

$$
[x, y]=\frac{i a^{2}}{\hbar} L_{z}
$$

This should be compared with Pryce [26] 27], formula (39) valid for systems with internal angular momentum. Snyder's idea is now a cornerstone of noncommutative geometry, the implications of which appears to be very important.

There is now a great deal of models with noncommutative coordinates that are obtained from different routes. As we have seen non-commutativity of coordinates is not linked to a breaking of the Lorentz invariance. In this paper we have concentrated ourselves in the problem of finding a correct set of quantum operators that satisfy Casalbuoni-Pryce relations for the superparticle (34]39). But the procedure used is

not restricted to this system. We show that non-commuting operators arise naturally when a set of commuting operators are projected onto a subspace.

\section{Acknowledgments}

This work was supported by Did-Usb grants Gid-30 and Gid-11 and by Fonacit grant G-2001000712. N.H thanks S.Okubo for bringing Ref. [18 to his attention.

\section{A Appendix}

In this final section we collect some formulas and conventions that are necessary in order to follow the calculations. Most conventions are taken from Wess and Bagger but not all.

We set $\sigma^{0}=\bar{\sigma}^{0}=I$ and the rest are like Wess and Bagger [28]. We always rise and lower indices with the second index (for example $D^{a}=\varepsilon^{a b} D_{b}$ ). We select $\varepsilon_{12}=-1$. An important identity is

$$
\sigma_{b \dot{a}}^{\nu} \bar{\sigma}^{\lambda \dot{a} a} \sigma_{a \dot{b}}^{\mu}=-\eta^{\nu \lambda} \sigma_{b \dot{b}}^{\mu}-\eta^{\mu \lambda} \sigma_{b \dot{b}}^{\nu}+\eta^{\mu \nu} \sigma_{b \dot{b}}^{\lambda}-i \varepsilon^{\nu \lambda \mu \alpha} \sigma_{\alpha b \dot{b}}
$$

We define

$$
\begin{aligned}
\sigma_{a}^{\mu \nu b} & =\sigma_{a \dot{a}}^{\mu} \bar{\sigma}^{\nu \dot{a} b}-\sigma_{a \dot{a}}^{\nu} \bar{\sigma}^{\mu \dot{a} b} \\
\bar{\sigma}^{\mu \nu \dot{a}} & =\bar{\sigma}^{\mu \dot{a} b} \sigma_{b \dot{b}}^{\nu}-\bar{\sigma}^{\nu \dot{a} b} \sigma_{b \dot{b}}^{\mu}
\end{aligned}
$$


From where we deduce

$$
\begin{gathered}
\sigma^{\mu \nu} \sigma^{\alpha}+\sigma^{\alpha} \bar{\sigma}^{\mu \nu}=-4 i \varepsilon^{\mu \nu \alpha \lambda} \sigma_{\lambda} \\
\sigma_{b \dot{a}}^{\nu} \bar{\sigma}^{\lambda \dot{a} a} \sigma_{a \dot{b}}^{\mu}-\sigma_{b \dot{a}}^{\mu} \bar{\sigma}^{\lambda \dot{a} a} \sigma_{a \dot{b}}^{\nu}=-2 i \varepsilon^{\nu \lambda \mu \alpha} \sigma_{\alpha b \dot{b}}
\end{gathered}
$$

Another two important identities

$$
\begin{gathered}
\sigma_{\beta} \bar{\sigma}_{\lambda}=-\eta_{\beta \lambda}+\frac{1}{2} \sigma_{\beta \lambda} \\
\bar{\sigma}^{\mu \dot{a} a} \bar{\sigma}_{\mu}{ }^{\dot{b} b}=-2 \varepsilon^{a b} \varepsilon^{\dot{a} \dot{b}}
\end{gathered}
$$

Covariant derivatives are given by

$$
\begin{gathered}
P_{\mu}=-i \partial_{\mu} \\
\Pi_{a}=-i \partial_{a} \quad \bar{\Pi}_{\dot{a}}=-i \bar{\partial}_{\dot{a}} \\
D_{a}=\partial_{a}+i \sigma^{\mu}{ }_{a \dot{b}} \bar{\Theta}^{\dot{b}} \partial_{\mu}=i \Pi_{a}-P_{\mu} \sigma^{\mu}{ }_{a \dot{b}} \bar{\Theta}^{\dot{b}} \\
\bar{D}_{\dot{b}}=-\bar{\partial}_{\dot{b}}-i \Theta^{a} \sigma^{\mu}{ }_{a b} \partial_{\mu}=-i \bar{\Pi}_{\dot{b}}+P_{\mu} \Theta^{a} \sigma^{\mu}{ }_{a \dot{b}}
\end{gathered}
$$

The supersymmetry charges

$$
\begin{gathered}
Q_{a}=\Pi_{a}-i P_{\mu} \sigma_{a \dot{a}}^{\mu} \bar{\Theta}^{\dot{a}} \\
\bar{Q}_{\dot{a}}=-\bar{\Pi}_{\dot{a}}+i P_{\mu} \sigma_{a \dot{a}}^{\mu} \Theta^{a} .
\end{gathered}
$$

Note that we always use a lower case letter for a classical observable and a capital letter for a quantum operator. In all cases an operator without any additional label behaves like in the Schrödinger representation. For example

$$
\begin{array}{cc}
X^{\mu} \Psi=x^{\mu} \Psi \quad \Theta^{a} \Psi=\theta^{a} \Psi \\
P_{\mu} \Psi=-i \partial_{\mu} \Psi \quad \Pi_{a} \Psi=-i \partial_{a} \Psi
\end{array}
$$

Projected operators are often indicated with a label $(A, C$ or $T)$. The momentum operator $P_{\mu}$ is an exception for this rule since we never write $P_{\mu}^{\mathrm{C}}$ for $\mathbb{P}_{\mathrm{C}} P_{\mu} \mathbb{P}_{\mathrm{C}}$. We think that this small abuse of notation cause no confusion.

Some useful commutators and anticommutators are [28]

$$
\begin{gathered}
{\left[\bar{D}^{2}, D^{a}\right]=-4 i \bar{\sigma}^{\mu \dot{b a}} \bar{D}_{\dot{b}} \partial_{\mu}} \\
{\left[\bar{D}^{\dot{a}}, D^{2}\right]=-4 i \bar{\sigma}^{\mu \dot{a} a} D_{a} \partial_{\mu}} \\
{\left[D^{2}, \bar{D}^{2}\right]=-8 i D \sigma^{\mu} \bar{D} \partial_{\mu}-16 \partial^{2}} \\
\left\{D^{2}, \bar{D}^{2}\right\}=16 \partial^{2}+2 \bar{D}_{\dot{a}} D^{2} \bar{D}^{\dot{a}} \\
{\left[D^{2}, X^{\mu}\right]=2 i D^{a} \sigma_{a \dot{a}}^{\mu}{ }_{a \dot{\Theta}}} \\
{\left[\bar{D}^{2}, X^{\mu}\right]=-2 i \Theta^{a} \sigma_{a \dot{a}}^{\mu} \bar{D}^{\dot{a}}}
\end{gathered}
$$


From theses we may deduce

$$
\begin{array}{cc}
{\left[\mathbb{P}_{\mathrm{T}}, \Theta^{a}\right]=\frac{1}{8 P^{2}}\left\{D^{a}, \bar{D}^{2}\right\}} \\
{\left[\mathbb{P}_{\mathrm{C}}, \Theta^{a}\right]=\frac{-1}{8 P^{2}} \bar{D}^{2} D^{a} \quad\left[\mathbb{P}_{\mathrm{C}}, \bar{\Theta}^{\dot{a}}\right]=\frac{-1}{8 P^{2}} \bar{D}^{\dot{a}} D^{2}} \\
{\left[\mathbb{P}_{\mathrm{A}}, \Theta^{a}\right]=\frac{-1}{8 P^{2}} D^{a} \bar{D}^{2}} & {\left[\mathbb{P}_{\mathrm{A}}, \bar{\Theta}^{\dot{a}}\right]=\frac{-1}{8 P^{2}} D^{2} \bar{D}^{\dot{a}}} \\
{\left[\bar{D}^{2}, \Pi_{b}\right]=2 i P_{\alpha} \sigma_{b \dot{b}}^{\alpha} \bar{D}^{\dot{b}}} & {\left[D^{2}, \bar{\Pi}_{\dot{b}}\right]=2 i P_{\alpha} \sigma_{b \dot{b}}^{\alpha} D^{b}}
\end{array}
$$

An important formula which can be found in [18]:

$$
\mathbb{P}_{\mathrm{T}}\left(\bar{D} \bar{\sigma}^{\mu} D\right) \mathbb{P}_{\mathrm{T}} P_{\mu}=-2 P^{2} \mathbb{P}_{\mathrm{T}}
$$

Note that $0=\left[X^{\mu}, 1\right]=\left[X^{\mu}, P^{2} / P^{2}\right]$ implies

$$
\left[X^{\mu}, \frac{1}{P^{2}}\right]=-\frac{2 i}{\left(P^{2}\right)^{2}} P^{\nu}
$$

The covariant derivatives act as interwinding operators

$$
\begin{array}{ll}
\mathbb{P}_{\mathrm{A}} D_{b}=D_{b} \mathbb{P}_{\mathrm{T}} & \mathbb{P}_{\mathrm{T}} \bar{D}_{\dot{a}}=\bar{D}_{\dot{a}} \mathbb{P}_{\mathrm{A}} \\
\mathbb{P}_{\mathrm{T}} D_{a}=D_{a} \mathbb{P}_{\mathrm{C}} & \bar{D}_{\dot{a}} \mathbb{P}_{\mathrm{T}}=\mathbb{P}_{\mathrm{C}} \bar{D}_{\dot{a}}
\end{array}
$$

\section{References}

[1] A. Salam and J. Strathdee, "Supergauge transformations" Nucl. Phys. B76, 477 (1974)

[2] R. Casalbuoni, "The classical mechanics for Bose-Fermi systems", Nuov. Cim. A33, N.3, 389-431. (1976).

[3] L. Brink and J.H. Schwarz, "Quantum superspace”, Phys. Lett. B100, 310 (1983)

[4] A.J. Hanson and T. Regge, "The relativistic spherical top", Ann. of Phys., 87, 498-566 (1974)

[5] M.B. Green and J. H. Schwarz, "Covariant description of superstrings", Phys. Lett., B136, 367 (1984)

[6] W. Siegel, "Hidden local supersymmetry in the supersymmetric particle action", Phys. Lett. B128, 391 (1983)

[7] J.A. de Azcárraga and J.Lukierski, "Supersymmetric particles with internal symmetries and central charges. ", Phys. Lett. 113B, (1982), 170; J.A. de Azcárraga and J.Lukierski, "Supersymmetric particles in N=2 Superspace:Phase space variables and hamiltonian dynamics .", Phys. Rev. Vol D28, (1983),1337. 
[8] A.S.Galperin E.A.Ivanov V.I.Ogievetsky,E.S.Sokatchev., "Harmonic Superspace", Cambridge University Press (2001)

[9] F.Delduc, A.Galperin and E.Sokatchev, "Lorentz Harmonic (Super)fields and (Super)particles"Nucl.Phys.B368, 143-174,1992]

[10] D.Sorokin, "Superbranes and superembeddings.", Phys.Rep.329, 1, (2000.

[11] N. Berkovits "Towards a covariant quatization of the supermembrane" JHEP, 09:51, 2002

[12] L. Lusana, "Relativistic mechanic with constraints and pseudoclassical models of supersymetry" in Supersymetry and supergravity 1983, Proceedings of the XIXth Winter School and Wokshop of theoretical Physics, Poland 14-26 (1983). World Scientific

[13] A. Frydryszak, " $N$-extended free superfields $(N=2,4,6,8)$ from quantization of a supersymmetric particle model", Phys. Rev. Vol D30, 2172-2180 (1984)

[14] R. Gianvitorio, A. Restuccia and J. Stephany, "On the quantization of field theories with second class constraints", Mod. Phys. Lett. A6 (1991) 2121.

[15] A. Restuccia and J. Stephany, "Gauge fixing in extended phase space and path integral quantization of systems with second class constraints " Phys. Lett. B305 (1993) 348.

[16] N.Hatcher, A. Restuccia, J. Stephany, "On the quantum mechanics of a supermembrane wrapped on a two circle" hep-th/0506042.

[17] M. Abou-Zeid, B. de Wit, D. Lüst, H. Nicolai, Phys. Lett., 466B, (1999), 144.

[18] S. Okubo, "Structure of superspace in supersymmetry", Phys. Rev. D29, 269-284 (1984)

[19] V. Rittenberg and E. Sokatchev, "Decomposition of extended superfields into irreducible representations of supersymmetry", Nuc. Phys. B193, 477-501 (1981).

[20] D. V. Volkov and A. I. Pashnev, "Supersymmetric lagrangian for particles in proper time", Theor. Math. Phys. 44 (1980), 770-773

[21] F. Constantinescu, "Definite and Indefinite Inner Product on Superspace", hep-th/0404182

[22] S.J. Gates, M.T. Grisaru, M. Rocek and W. Siegel, "One thousand and one lesons in supersymmetry", Frontiers in physics v.58, Benjamin, (1983)

[23] A. Salam and J. Strathdee, "On superfields and Fermi-Bose symmetry", Phys. Rev. D11, 1521-1535, (1975) 
[24] E. Sokatchev, "Projection operators and supplementary conditions for superfields with arbitrary spin", Nuc Phys B99, 96-108 (1975)

[25] H. S. Snyder, "Quantized Space-Time”, Phys. Rev. 71, 38-41, 1947

[26] M. H. L. Pryce, "The mass-centre in the restricted theory of relativity and its connexion with the quantum theory of elementary particles", Proc. Roy. Soc. of London A195, 62-81 (1948)

[27] T.D. Newton and E. P. Wigner, "Localized states for elementary systems", Rev. Mod. Phys., 21, 400-406 (1949).

[28] J. Wess and Bagger, "Supersymmetry and supergravity", Princeton Series in Physics (1982) 